\title{
Variability of the HeI5876 $\AA$ line in early type chemically peculiar stars
}

\author{
G. Catanzaro ${ }^{1,4}$, F. Leone L $^{2,4}$, and F.A. Catalano ${ }^{3,4}$ \\ 1 Dipartimento di Fisica della Materia e Tecnologie Fisiche Avanzate, Università di Messina, Salita Sperone 31, I-98166 Messina, \\ Italy \\ e-mail: gcatanzaro@alpha4.ct.astro.it \\ 2 Osservatorio Astrofisico di Catania, Città Universitaria, Viale A. Doria 6, I-95125 Catania, Italy \\ e-mail: fleone@alpha4.ct.astro.it \\ ${ }^{3}$ Istituto di Astronomia, Università di Catania, Città Universitaria, Viale A. Doria 6, I-95125 Catania, Italy \\ e-mail: fcatalano@alpha4.ct.astro.it \\ ${ }^{4}$ C.N.R.-G.N.A., UdR di Catania, Città Universitaria, Viale A. Doria 6, I-95125 Catania, Italy
}

Received June 3; accepted August 3, 1998

\begin{abstract}
Chemically peculiar stars present spectral and photometric variability with a single period. In the oblique rotator model, the non homogeneous distribution of elements on the stellar surface is at the origin of the observed variations. As to helium weak stars, it has been suggested that photometric and helium line equivalent width variations are out of phase. To understand the behaviour of helium in CP stars, we have obtained time resolved spectra of the HeI5876 $\AA$ line for a sample of 16 chemically peculiar stars in the spectral range B3 - A1 and belonging to different sub-groups.

The HeI5876 $\AA$ line is too weak to be measured in the spectra of the stars HD 24155, HD 41269, and HD 220825. No variation of the equivalent width of the selected He line has been revealed in the stars HD 22920, HD 24587, HD 36589, HD 49606, and HD 209515. The equivalent width variation of the HeI5876 A line is in phase with the photometric variability for the stars HD 43819 , HD 171247 and HD 176582. On the contrary it is out of phase for the stars HD 28843, HD 182255 and HD 223640. No clear relation has been found for the stars HD 26571 and HD 177003.
\end{abstract}

Key words: stars: chemically peculiar - stars: abundances — stars: early type

Send offprint requests to: G. Catanzaro - Istituto di Astronomia, Università di Catania, Viale A. Doria 6, I-95125 Catania, Italy.

\section{Introduction}

Among the early type stars of the main sequence various groups of chemically peculiar stars (henceforth CP stars) are found. According to the General Catalogue of Ap and Am stars (Renson et al. 1991), among the 6684 so far known or suspected CP stars, more than half (3427) are Am stars (CP1) the remaining 3257 being Bp or Ap. Among these only 190 stars belong to the He strong (82) or He weak (108) subgroups. Usually helium is underabundant in the coolest CP stars and overabundant in the hottest ones.

CP stars are characterised by spectral and photometric variations with a common period. In the oblique rotator model, proposed by Stibbs (1950), chemical elements are not homogeneously distributed on the stellar surface and the observed variations are due to the stellar rotation. Studying the photometric variability of six helium weak stars, Catalano \& Leone (1996) found that the equivalent width of the HeI4026 $\AA$ varies out of phase with respect to the photometric variability.

To investigate the behavior of helium and its relation to the spectral and light variability, we have performed time resolved spectroscopy of the HeI5876 A line for a sample of $16 \mathrm{CP}$ stars in the B6-A1 spectral range and belonging to different peculiarity classes.

\section{Observations and data analysis}

For the chemically peculiar stars listed in Table 1 echelle spectra were obtained in 1995 at the $2.1 \mathrm{~m}$ telescope of the Complejo Astronómico El Leoncito equipped with a Boller \& Chivens cassegrain spectrograph and in 1997 at the 
Table 1. Average equivalent width $\langle\mathrm{EW}\rangle$ and relative standard deviation $\sigma$ for the observed chemically peculiar stars. Spectral type and peculiarity class are from The General Catalogue of Ap and Am stars by Renson et al. (1991). $T_{\text {eff }}$ and log $g$ have been computed according to Moon \& Dworetsky (1985) and Napiwotzki et al. (1993). $N$ refers to the number of spectra, $P(\mathrm{~d})$ to the variability period in days with the relative reference. Source for $V$ magnitudes and $v_{\mathrm{e}} \sin i$ was SIMBAD database. The "no line" remark indicates the absence of the HeI5876 $\AA$ line in the spectrum; "constant", the absence of EW variations; "in phase", the coincidence of maxima of the $E W$ and photometric variations; and "out of phase", the coincidence of maxima of the $E W$ variation with the minima of photometric variations

\begin{tabular}{|c|c|c|c|c|c|c|c|c|c|c|c|c|}
\hline HD & $\mathrm{HR}$ & Sp. Type & $V$ & $v_{\mathrm{e}} \sin i$ & $T_{\text {eff }}$ & $\log g$ & $N$ & $\langle E W\rangle$ & $\sigma$ & Remarks & $P(\mathrm{~d})$ & Reference \\
\hline 22920 & 1121 & $\overline{\mathrm{B} 8 \mathrm{Si}}$ & $\overline{5.53}$ & 120 & $\overline{13700}$ & 3.72 & $\overline{8}$ & $\overline{125}$ & $\overline{13}$ & constant & & \\
\hline 24155 & 1194 & B9 Si & 6.30 & 50 & 13700 & 3.96 & 9 & & & no line & & \\
\hline 24587 & 1213 & B6 & 4.65 & 40 & 14100 & 4.23 & 18 & 325 & 20 & constant & & \\
\hline 26571 & 1297 & B8 Si & 6.12 & 29 & 13000 & 3.16 & 18 & 145 & 15 & & 1.0646 & Winzer (1974) \\
\hline 28843 & 1441 & B9 He wk & 5.81 & $70^{*}$ & 15300 & 4.17 & 21 & 70 & 20 & out of phase & 1.373813 & Mathys et al. (1986) \\
\hline 36589 & 1860 & B7 & 6.18 & 90 & 14000 & 3.97 & 9 & 350 & 8 & constant & & \\
\hline 41269 & 2139 & B9 Si & 6.20 & 75 & 10800 & 3.82 & 10 & & & no line & & \\
\hline 43819 & 2258 & B9 Si & 6.30 & 14 & 11100 & 3.66 & 12 & 95 & 30 & in phase & 15.0305 & Adelman (1997) \\
\hline 171247 & 6967 & B8 Si & 6.42 & 60 & 11300 & 3.40 & 23 & 95 & 15 & in phase & 3.9124 & North (1992) \\
\hline 176582 & 7185 & B5 He wk & 6.41 & 145 & 18300 & 4.31 & 12 & 360 & 30 & in phase & 1.58175 & This work \\
\hline 177003 & 7210 & $\mathrm{~B} 3 \mathrm{He}$ & 5.37 & 40 & 18700 & 4.11 & 14 & 705 & 25 & & 1.835 & This work \\
\hline 182255 & 7358 & B6 He wk & 5.13 & 45 & 14400 & 4.17 & 18 & 325 & 15 & out of phase & 1.26263 & This work \\
\hline 209515 & 8407 & A0 CrSiMg & 5.60 & 100 & 9600 & 3.73 & 7 & 40 & 5 & constant & & \\
\hline 220825 & 8911 & $\mathrm{~A} 1 \mathrm{CrSrEu}$ & 4.94 & 30 & 10400 & 4.46 & 7 & & & no line & & \\
\hline 223640 & 9031 & B9 SiSrCr & 5.19 & 20 & 12400 & 3.62 & 6 & 60 & 15 & out of phase & 3.735239 & North et al. (1992) \\
\hline
\end{tabular}

*The projected rotational velocity of HD 28843 has been here measured.

$91 \mathrm{~cm}$ telescope of the Catania Astrophysical Observatory equipped with a Czerney-Turner echelle spectrograph.

The data were analysed the using IRAF package. The lines of the wavelength calibration lamp show that $R=16000$ for the 1997 data set and $R=13000$ for the 1995 data set. The achieved $\mathrm{S} / \mathrm{N}$ was between 100 and 200. When possible, equivalent widths were measured by a Gaussian fit of spectral lines after a possible continuum slope; otherwise a measure of the area between the line profile and the continuum was obtained. Following Leone et al. (1995), we estimated the error in the measured equivalent width with the relation:

$\Delta W=\frac{1}{2}\left(2 \frac{v_{\mathrm{e}} \sin i}{c} \lambda\right) \frac{1}{S / N}$

where the quantity in brackets is the total extension of the line as deduced from the rotational broadening. Adopted $v_{\mathrm{e}} \sin i$ values (Table 1 ) are from SIMBAD with the exception of HD 28843 whose projected rotational velocity was measured from the unblended SirI5865 ^ line.

The initial ephemeris of program stars were taken from Catalano \& Renson (1984, 1988, 1997), and Catalano et al. (1991, 1993), and references therein. If necessary, periods were established using our spectral observations and Hipparcos photometry ${ }^{1}$. A least squares fit of measured $E W$ 's and $\mathrm{H}_{\mathrm{p}}$ magnitudes has been performed by adopting the function:

\footnotetext{
1 The Hipparcos filter, referred to as $\mathrm{Hp}$, extends from $3550 \AA$ to $8900 \AA$ with the maximum at $4350 \AA$. The typical accuracy of Hipparcos measurements, at the 8th magnitude, is given as $0.0015 \mathrm{mag}$ (ESA, 1997).
}

$$
\begin{aligned}
A_{0}+A_{1} \sin \left(2 \pi\left(t-t_{0}\right) / P+\phi_{1}\right) & \\
& +A_{2} \sin \left(4 \pi\left(t-t_{0}\right) / P+\phi_{2}\right)
\end{aligned}
$$

where $t$ is the JD date, $t_{0}$ is the assumed initial epoch, $P$ is the period in days. A sine wave and its first harmonic appear to be quite adequate functions to describe the light curves and the spectral variations (North 1984; Mathys \& Manfroid 1985). The error in the period value has been evaluated according to the relation given in Horne \& Baliunas (1986).

As to the coolest CP stars, the effective temperatures and gravities have been determined by means of ad hoc Napiwotzki et al. (1993) relations. As to helium peculiar stars, Hauck \& North (1993) found that classical methods are still reliable to determine their effective temperature. Thus, we have used the Moon \& Dworetsky (1985) grids as coded by Moon (1985). The source of Strömgren photometry was SIMBAD.

To ascertain if the selected stars present a peculiar helium abundance we have compared the measured equivalent widths of the HeI5876 $\AA$ line with the NLTE computations of Leone \& Lanzafame (1998) for solar composition stars with $\log g=3.5,4.0$ and 4.5 and $9000 \mathrm{~K}$ $<T_{\text {eff }}<19000 \mathrm{~K}$.

\section{Individual stars}

\subsection{HD 22920 $(=H R 1121=22 \mathrm{Eri})$}

According to Maitzen (1976), the silicon star HD 22920 has a low value of the photometric peculiarity index $\Delta a$ 
$(=0.011)$. Photometric observations have been carried out by Bartholdy (1988) who found this star to be variable with a period of $3.95 \mathrm{~d}$. North (1990 priv. comm.) found two possible periods almost equally probable: $3.96 \mathrm{~d}$, very close to Bartholdy's (1988), and $1.33 \mathrm{~d}$.

No evidence of variability has been found in our spectra of the HeI5876 $\AA$ line. The mean value of the equivalent width is: $\langle E W\rangle=125 \pm 13 \mathrm{m \AA}$. The effective temperature of HD 22920 resulting from Napiwotzki et al. (1993) relation is $T_{\text {eff }}=13700 \mathrm{~K}$. Figure 9 shows that the HeI5876 $\AA$ line equivalent width of HD 22920 is smaller than expected for a main sequence star of the same effective temperature.

\subsection{HD $24155(=H R 1194=$ V766 Tau $)$}

The $U B V$ photometric variability of HD 24155 has been studied by Winzer (1974), who reported a possible period of 2.5352 d. Renson \& Manfroid (1981) found $P=2.53465 \pm 0.00015 \mathrm{~d}$. The observed light curves show a quite large amplitude (0.10 mag) with very sharp minima and quite broad maxima, hence this star is the fourth largest amplitude silicon star known, exceeded only by HD 215441, CU Vir and HR 7058.

Assuming Renson \& Manfroid's (1981) period, our nine spectra are well distributed in phase, but none of them shows a measurable HeI5876 A line. Because of its effective temperature of $13700 \mathrm{~K}$, HD 24155 is an extremely helium weak star (Fig. 9).

\section{3. $H D 24587\left(=H R 1213=\tau^{8}\right.$ Eri)}

HD 24587 is listed in the General Catalogue of Ap and Am stars by Renson et al. (1991) as a suspected CP star. Feinstein (1978) used this star as standard for his measurements of hydrogen lines in He weak stars. HD 24587 has in fact been considered as a standard for uvby (Garnier 1972 - personal communication to Mathys et al. 1986) and $\beta$ photometry (Strauss \& Ducati 1981). Mathys et al. (1986) found this star to be a light variable with a period of $1.728 \mathrm{~d}$ and concluded that the light curves resemble those of many CP stars. Recently Leone \& Catanzaro (1998) have performed a spectroscopic study and concluded that this star presents chemical elements which are slightly underabundant with respect to main sequence stars.

Our measurements of the HeI5876 $\AA$ line do not show any variation of the equivalent width; the mean value is: $\langle E W\rangle=325 \pm 20 \mathrm{~mA}$. From Moon's algorithm we find that $T_{\text {eff }}=14100 \mathrm{~K}$ and the $E W$ of the HeI5876 $\AA$ line is close to the value expected for a main sequence star (Fig. 9). These facts confirm Leone \& Catanzaro's (1998) conclusion that HD 24587 is not a peculiar star.

\subsection{HD 26571 (= HR 1297)}

The peculiarity of HD 26571 was first noted by Gulliver (1971) and independently confirmed by Bond (1972). On the basis of his spectra, Gulliver (1971) described this star as a spectrum variable. Photometric observations were obtained by Winzer (1974), who found HD 26571 to vary with a period of $1.0646 \mathrm{~d}$.

Figure 1 shows the $E W$ variation of the HeI5876 $\AA$ line versus the phase computed assuming the initial epoch coincident with the light maximum as given by Winzer (1974):

$\mathrm{JD}(U B V \max )=2441246.81+1.0646 \mathrm{E}$.

Winzer (1974) has not published the uncertainty in the period determination, hence we have estimated the error by applying the Horne \& Baliunas (1986) relation to our data and have found that equivalent widths are phased with an expected error $\Delta \Phi=0.3$. This means that no phase relation can be determined between the photometric and our spectral variations.

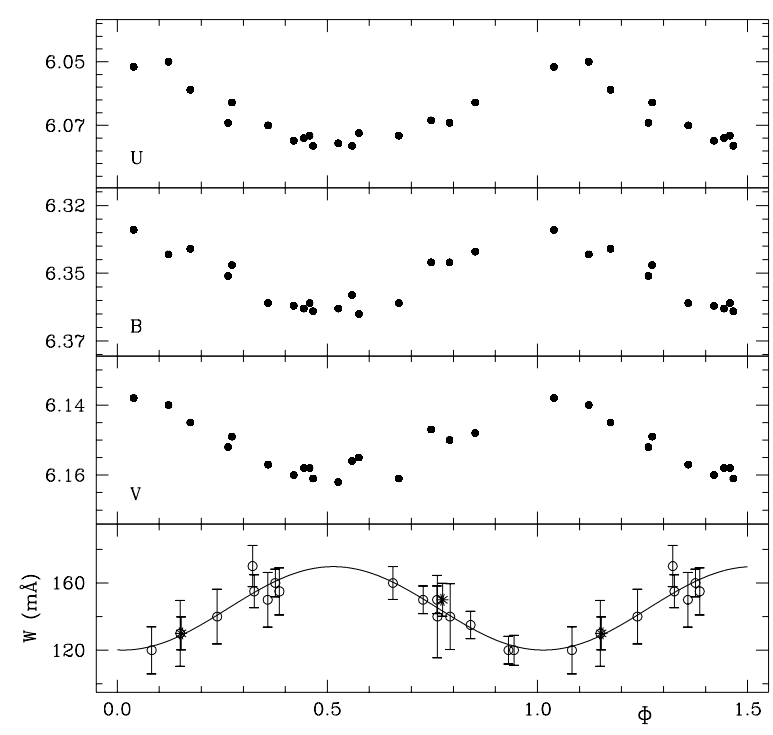

Fig. 1. Equivalent width variations of HD 26571. The errorbar is equal to the error in the equivalent width as given by Eq. (1). Photometric data are from Winzer (1974). Phases have been computed by Eq. (3). Open circles refer to the 1997 data, stars to the 1995 data. The fitting of $E W$ 's performed by Eq. (2) is reported with a solid line. The phase relation between $E W$ and photometric variations may be not real because of the large time gap between both data sets and the relatively low accuracy of the variability period

\subsection{HD $28843(=H R 1441=D Z$ Eri $)$}

HD 28843 was classified as B9IV Si He-wk by Davis (1977) and it is classified as B9 He wk in the General 
catalogue of $A p$ and Am Stars. The photometric variability of HD 28843 had been detected for the first time by Cousins \& Stoy (1966) while its peculiar character had been confirmed by Jaschek et al. (1969). Photometric observations of this star have been carried out by Pedersen \& Thomsen (1977) who found variability with a period of $1.374 \pm 0.006 \mathrm{~d}$. This value was improved by Pedersen (1979) to the value $1.37375 \pm 0.00035 \mathrm{~d}$. Manfroid et al. (1984) also used Pedersen \& Thomsen's (1977) data to improve the period, their most probable value being 1.373813 $\pm 0.000012 \mathrm{~d}$. Mathys et al. (1986) concluded that the ambiguity in the choice of the best peak in the periodogram could be removed by inclusion of the measurements of Dean (1980), confirming the value obtained by Manfroid et al. (1984). Further photometric observations have been carried out by Waelkens (1985), by the team of the ESO Long-Term Photometry of Variable Project (Manfroid et al. 1994; Sterken et al. 1995), and by the team of Hipparcos (ESA, 1997).

Our spectroscopic data are plotted in Fig. 2, versus the phase computed from the ephemeris elements of Mathys et al. (1986):

$\mathrm{JD}(u v b y \max )=2442777.5+1.373813 E$.

The amplitude of the equivalent line width variations is of the order of $75 \mathrm{m \AA}$. From Fig. 2 a clear anti-correlation is evident between the HeI5876 A equivalent line width and all the Hipparcos and uvby light curves, in the sense that light minima occur at the phase of maximum HeI. Because of the period error determined by Manfroid et al. (1984), the expected phase error in our $E W$ variations is $\Delta \Phi=$ 0.03. $E W$ variations of the HeI5876 $\AA$ line are then out of phase with respect to light variations.

Even if most of our equivalent widths periodically vary with the ephemeris computed with Eq. (4), we have found several (5 out of 21) spectra where the HeI5876 line is absent (Fig. 2).

\section{6. $H D 36589(=H R$ 1860)}

In the General Catalogue of Ap and Am stars, the star HD 36589 is a suspected CP star. Bossi \& Guerrero (1989) and Hao et al. (1996) have used it as a comparison star for photometric observations. Leone \& Catanzaro (1998) derived chemical abundances and found that HD 36589 shows nearly solar values and no evidence of spectral variability.

From our spectra we confirm this result: no evidence of variation is found in the Her5876 $\AA$ equivalent line width. On the hypothesis that HD 36589 is not a CP star, we have determined $T_{\text {eff }}=14000 \mathrm{~K}$ by mean of Moon's relations and found that the average value of the equivalent widths $(\langle W\rangle=350 \pm 8 \mathrm{m \AA})$ is very close to that of normal main sequence stars of the same spectral type (Fig. 9).

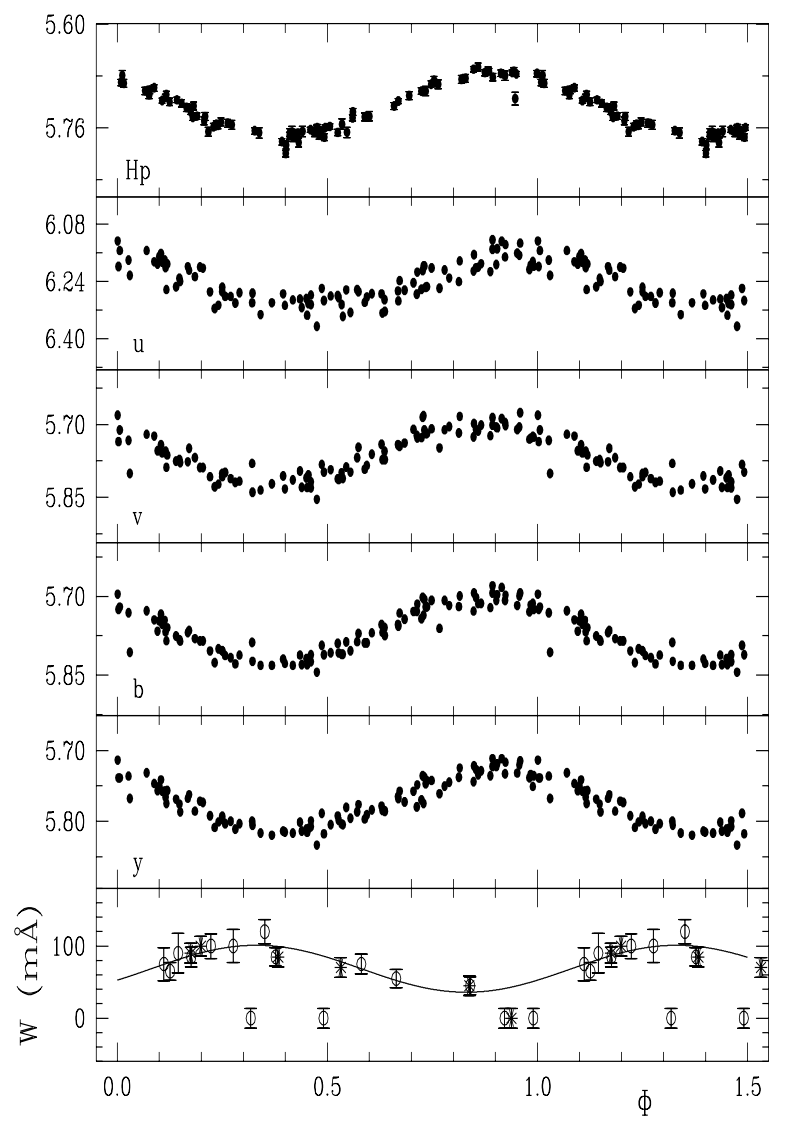

Fig. 2. Equivalent width variations of HD 28843. The bar is equal to the error in the equivalent width as given by Eq. (1). Open circles refer to the 1997 data, stars to the 1995 data. Photometric observations are from Hipparcos (ESA, 1997) and from the ESO LTPV Project (Manfroid et al. 1994; Sterken et al. 1995)

\section{7. $H D 41269(=H R 2139)$}

This star has been classified as B9p by Cowley et al. (1969) who described it as a mild silicon star. On the basis of a single observing run in $U B V$, Winzer (1974) found a period of $1.68 \mathrm{~d}$, although he could not rule out the resonance period of $2.47 \mathrm{~d}$, because of the few observed points.

In our spectra the HeI5876 $\AA$ line is too weak to be measured. According to Napiwotzki et al. (1993) relation, $T_{\text {eff }}=10800 \mathrm{~K}$. Figure 9 shows that the helium abundance is lower than the expected value for a main sequence star of this temperature.

\subsection{HD $43819(=H R 2258=H I P 30019=V 1155$ Ori $)$}

Cowley (1972) classified this star as B9IIIp Si. Photometric measurements of HD 43819 were performed in the $U B V$ system by Winzer (1974) who found a light variation with a period of $1.0785 \mathrm{~d}$. Later on Maitzen 


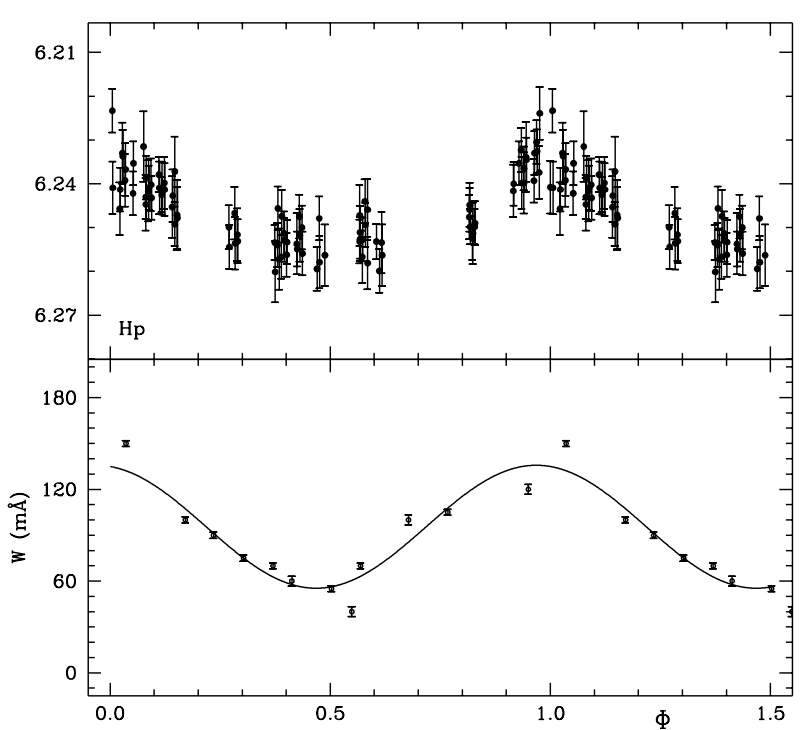

Fig. 3. Equivalent width variations of HD 43819. Errorbar is equal to the error in the equivalent width as given by Eq. (1). Photometric data are from Hipparcos (ESA, 1997)

(1980) found the light variation to occur with two possible periods: $0.93 \mathrm{~d}$ and $1.077 \mathrm{~d}$. A spectroscopic study of this sharp-lined star $\left(v_{\mathrm{e}} \sin i=14 \mathrm{~km} \mathrm{~s}^{-1}\right.$, Wolff \& Preston 1978) was carried out by Lopez-Garcia \& Adelman (1994), who found iron peak elements ten times overabundant and rare earths 1000 times overabundant with respect to solar values. From photometric uvby observations Adelman (1997) has deduced a period of $15.0305 \pm 0.0003 \mathrm{~d}$, longer than Winzer's (1974) and more consistent with the low rotational velocity of this star. This period is also confirmed by the Hipparcos observations (Fig. 3).

Our HeI5876 $\AA$ equivalent line widths are plotted in Fig. 3 versus the phase computed by means of Adelman's (1997) ephemeris elements:

$\mathrm{JD}(U \max )=2441254.16+15.0305 E$.

The observed $E W$ variation has an amplitude of the order of $80 \mathrm{~mA}$. From Fig. 3 we see a clear in-phase correlation between light and spectral variations. This correlation is expected to be real, the phase error being $\Delta \Phi \sim 0.01$.

\section{9. $H D 49606(=H R 2519=33 \mathrm{Gem})$}

The star HD 49606 is classified as a B8HgMnSi star by Renson et al. (1991). Photometric observations of HD 49606 were performed by Chunakova et al. (1981), who found the light variations to occur with a period of $3.099 \mathrm{~d}$, and by Glagolevskii et al. (1985), who found two possible period values, namely $3.3546 \mathrm{~d}$ and $1.41864 \mathrm{~d}$.

The HeI5876 ̊̊ equivalent line width observed in our spectra does not show any detectable variation, so that we consider this line does not vary with time. The average equivalent width is $\langle W\rangle=145 \pm 7 \mathrm{m \AA}$. This result confirms the one obtained by Hubrig \& Launhardt (1993)

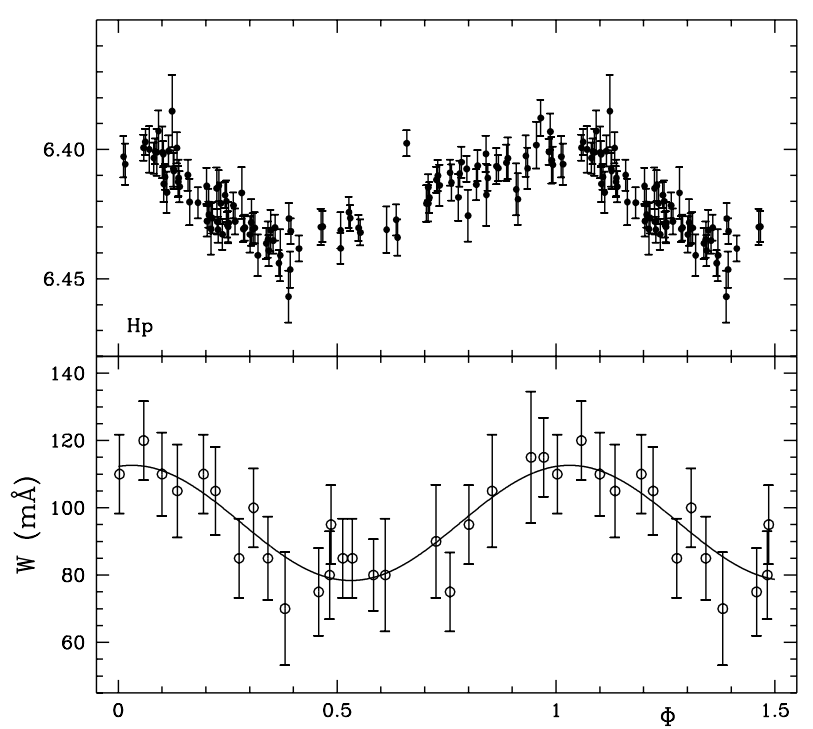

Fig. 4. Equivalent width variations of HD 171247. the bar is equal to the error in the equivalent width as given by Eq. (1). Photometry is from Hipparcos (ESA, 1997)

who searched for variations in the equivalent width of helium and some metallic lines and did not find any evidence of variability.

According to an elemental abundances analysis performed by Adelman et al. (1996) our observations show that helium is underabundant with respect to solar composition (Fig. 9).

\subsection{HD 171247 $(=$ HR $6967=H I P 90971)$}

The photometric variability of this star was detected by North (1992), who found the period to be $3.9124 \mathrm{~d}$. This value of the period is confirmed by the Hipparcos photometry (1997) which gives an error on the period equal to $0.0004 \mathrm{~d}$ applying Horne \& Baliunas (1986) formula.

Computing the phase of the measured equivalent widths by means of North's (1992) ephemeris:

$\mathrm{JD}([U]$ Geneva $\max )=2447178.245+3.9124 E$

we find a sinusoidal variation of the HeI5876 $\AA$ line strength (Fig. 4) with an amplitude of the order of $45 \mathrm{m \AA}$.

Converting the period error to a phase error, we get $\Delta \Phi=0.07$. We can thus conclude that $H_{\mathrm{p}}$ and $E W$ variability are in phase for the silicon star HD 171247.

\subsection{HD 176582 $(=H R 7185=H I P 93210)$}

This star is classified as a silicon star (Renson et al. 1991). Spectroscopic observations of the HeI4026 A line strength were carried out by Pedersen (1976), who found a variation with the period $0.8143 \mathrm{~d}$. The period is not representative of the variability of Hipparcos photometry and HeI5876 equivalent width. 


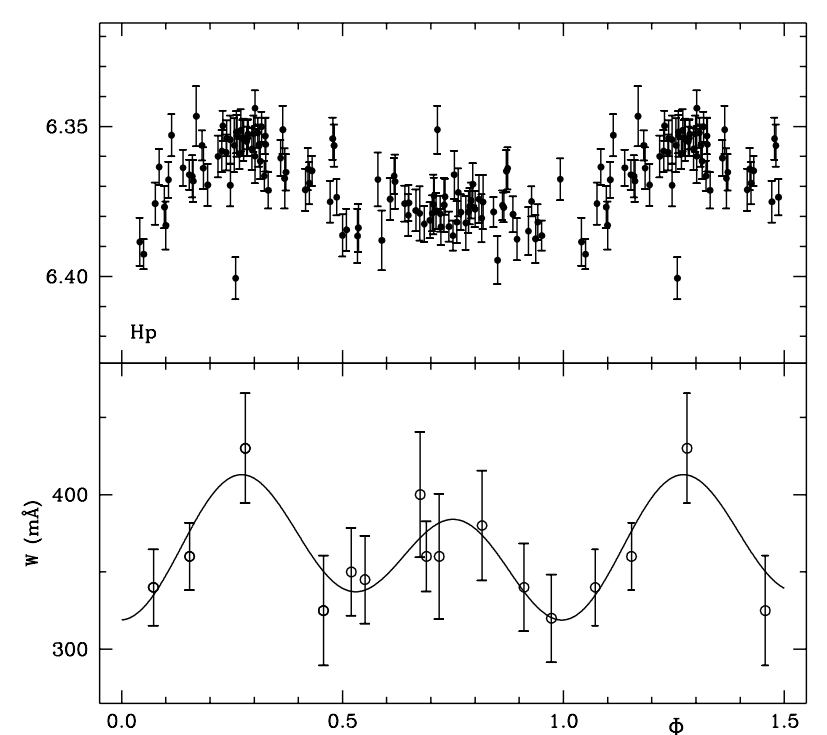

Fig. 5. Equivalent width variations of HD 176582. Errorbar is equal to the error in the equivalent width as given by Eq. (1). Photometry is from Hipparcos (ESA, 1997)

By using our spectroscopic data and Hipparcos photometry we found a period of $1.5817 \pm 0.0003 \mathrm{~d}$. The observations are plotted in Fig. 5 versus the phase computed by means of the ephemeris elements:

$\mathrm{JD}(E W \min )=2450624.6410+1.5817 E$.

From this figure we see that both curves show a clear evidence of a double-wave variation. The observed $E W$ amplitude is of the order of $40 \mathrm{m \AA}$. The expected phase error is $\Delta \Phi=0.05$, and the Hipparcos photometry appears to vary in phase with the equivalent width variations of the HeI5876 ̊ line.

\subsection{HD $177003(=H R 7210=H I P$ 93299)}

Schöneich \& Zelwanowa (1984) from their photometric observations in the UV filters found two possible periods: $0.66 \mathrm{~d}$ and $2.1 \mathrm{~d}$. From $U B V R I$ photometric observations, Vetö (1993) found this star to be light variable with a period of $0.724 \mathrm{~d}$ and amplitudes of about $0.1 \mathrm{mag}$ in all filters. An analysis of Hipparcos photometric data does not give a clear variability period.

Our spectroscopic data are not consistent with the periods given in the literature. A period search of our data yelds two possible values: $1.835 \pm 0.004 \mathrm{~d}$ and $2.186 \pm$ $0.005 \mathrm{~d}$. The HeI $5876 \AA$ line equivalent width variation is plotted in Fig. 6 versus the phase computed by means of the ephemeris elements:

$\mathrm{JD}(E W \max )=2450629.4099+1.835 E$

where we have adopted the shorter value of the period which has a smaller $\chi^{2}$ value. The variation shown in Fig. 6 has an amplitude of the order of $75 \mathrm{~m} \AA$. The photometric

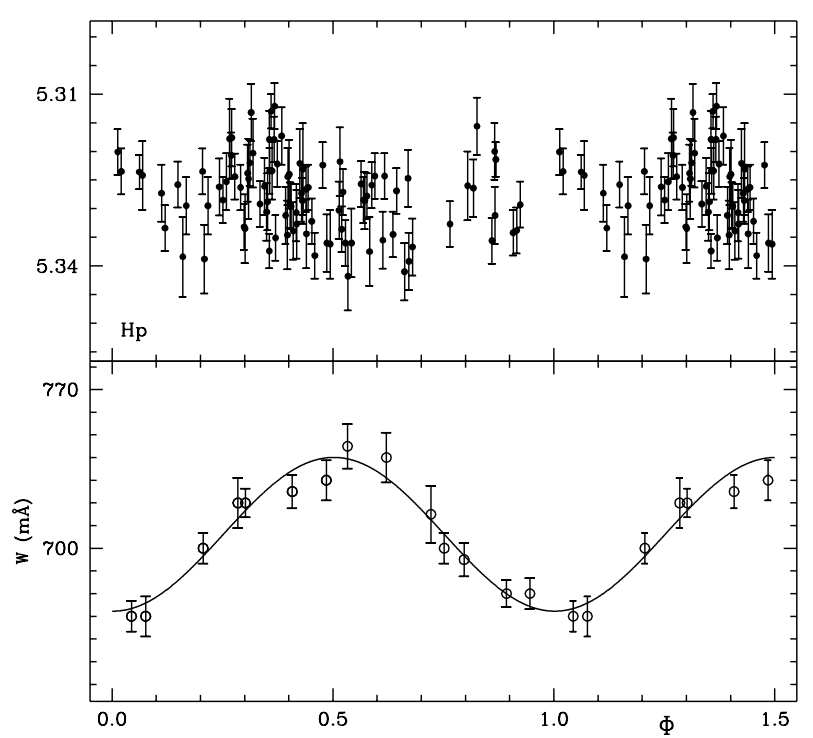

Fig. 6. Equivalent width variations of HD 177003. Errorbar is equal to the error in the equivalent width as given by Eq. (1)

variability is not clear for the $H_{\mathrm{p}}$ filter assuming this period (Fig. 6) and no conclusion can be drawn concerning a possible phase relation between photometric and spectral variations of the HeI5876 $\AA$ line.

\subsection{3. $H D 182255(=H R 7358=H I P 95260=3 \mathrm{Vul})$}

According to Hube \& Aikman (1991) this star is a nonradial pulsator. It has also been observed by Hipparcos, from whose photometry a period of $1.26239 \mathrm{~d}$ has been derived. However this value of the period is not perfectly consistent with our spectroscopic observations; instead, by using both sets of data the most probable value appears to be $1.26263 \pm 0.00005 \mathrm{~d}$. Adopting this period, the measured $E W$ of the HeI5876 $\AA$ line are plotted in Fig. 7 versus the phase computed by means of the ephemeris elements:

$\mathrm{JD}(E W \max )=2450650.4729+1.26263 E$.

The observed $E W$ amplitude is of the order of $65 \mathrm{~mA}$. Since the period error corresponds to a phase error $\Delta \Phi=0.09$, the reported out of phase relation between photometric and helium line variations is expected to be real (Fig. 7).

\subsection{HD 209515 (=HR $8407=$ V1942 Cyg)}

This star was classified as A0p by Osawa (1965) and as A0 IV by Cowley et al. (1969). From his photometric observations, Winzer (1974) found a period of $0.63703 \mathrm{~d}$, concluded that the observed photometric variation is typical for a silicon star and suggested that the correct classification should be A0p Si.

The equivalent width of the HeI5876 $\AA$ line of the cool CP star HD 209515 is constant: $40 \pm 5 \mathrm{m \AA}$. This value of 


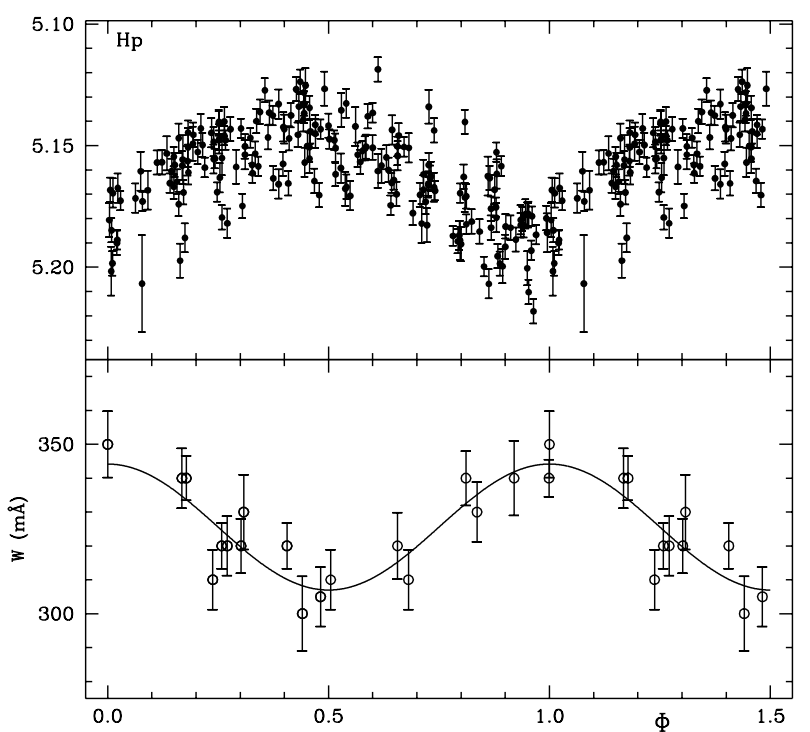

Fig. 7. The light and HeI $5876 \AA$ A equivalent line width variations of HD 182255. Errorbar is equal to the error in the equivalent width as given by Eq. (1). Photometry is from Hipparcos (ESA, 1997)

equivalent width is consistent with the helium abundance of a main sequence star (Fig. 9).

\subsection{HD 220825 $(=H R 8911=\kappa P s c)$}

The variability of HD 220825 had been detected for the first time by Rakosch (1962) who found a period of 0.5805 d. Recently, Ryabchikova et al. (1996) determined the period to be $1.418 \mathrm{~d}$ and magnetic observations performed by Borra \& Landstreet (1980) are also consistent with this value.

The HeI $\lambda 5876 \AA$ line is too weak to be measured. Assuming $T_{\text {eff }}=10400 \mathrm{~K}$, Fig. 9 shows that helium is underabundant in HD 220825 with respect to main sequence stars.

$$
\begin{aligned}
& \text { 3.16. HD 223640 }(=H R 9031=H I P 117629=108 \mathrm{Aqr} \\
& =E T \text { Aqr })
\end{aligned}
$$

The photometric variability of this star has been studied by several authors. Morrison \& Wolff (1971) found HD 223640 to be variable in the Strömgren system with a period of $3.73 \mathrm{~d}$ and noted that light curves show quite the same behaviour in all filters. Spectroscopic observations were carried out by Megessier \& Garnier (1972) who found strongly variable the $\mathrm{Ti}$ and $\mathrm{Sr}$ lines and constant the Fe lines. Moreover the Ti lines correlate with photometric variations in the sense that $\mathrm{Ti}$ lines are strongest when the star is brightest. This correlation has been interpreted by Megessier $(1974,1975)$ in terms of the oblique rotator model taking also into account the sign changes

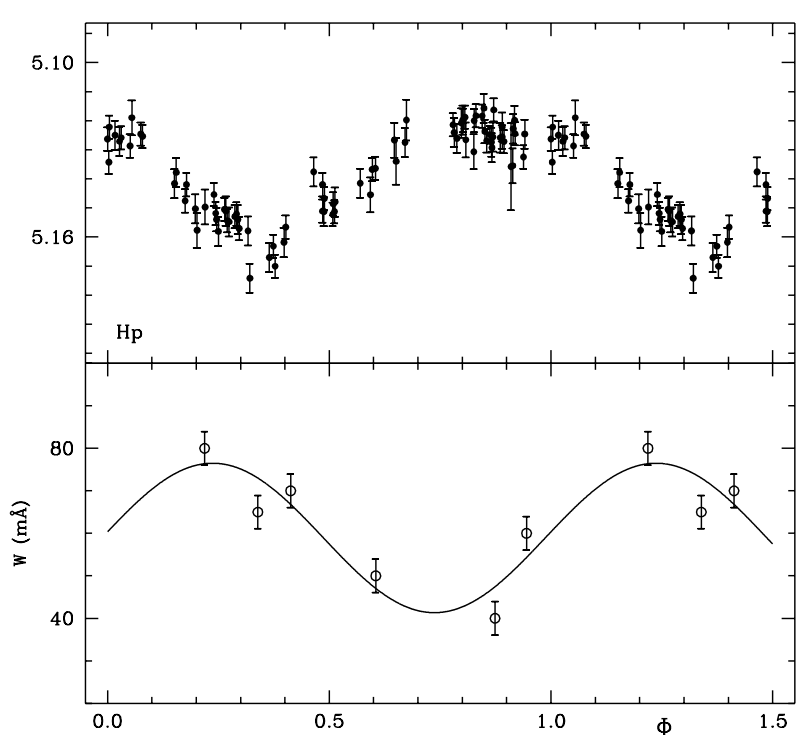

Fig. 8. Equivalent line width variations of HD 223640. Errorbar is equal to the error in the equivalent width as given by Eq. (1). Photometry is from Hipparcos (ESA, 1997)

of the magnetic field measurements by Babcock (1958). Photometric observations in the Geneva system have been performed by North et al. (1992); they found a period of $3.735239 \pm 0.000024 \mathrm{~d}$ which is consistent with the magnetic data. This period has been confirmed by photometric observations in the uvby system performed by Adelman \& Knox (1994) and Adelman (1997).

According to North et al. (1992), we phased the measured equivalent widths of the HeI5876 $\AA$ line by means of the ephemeris elements:

$\mathrm{JD}(u v b y \max )=2444696.820+3.735239 \mathrm{E}$.

The period uncertainty corresponds to a phase error $\Delta \Phi=0.004$. There is evidence of an anti-correlation between light and spectroscopic curves. The HeI line is strongest in coincidence with the light minimum: the helium distribution on the surface of HD 223640 is then not coincident with the $\mathrm{Ti}$ distribution.

\section{Conclusion}

In this paper we have presented spectroscopic observations of the HeI5876 $\AA$ line in $16 \mathrm{CP}$ stars (see Table 1). In the case of HD 26571, HD 28843, HD 43819, HD 171247 and HD 223640 the literature period values are accurate enough to represent our observations quite well. No variability has been detected in the stars HD 22920, HD 24587 , HD 36589, HD 49606, and HD 209515, while the HeI5876 ̊ line has been found to be too weak to be measured in the stars HD 24155, HD 41269 and HD 220825. In the case of the remaining stars, ie. HD 176582, 177003, and HD 182255, we have refined the value of the period by using both our own and literature data, when available. 
In the attempt to study the phase correlations between light and spectral variations, we have calculated the error on phase. From these calculations we can see that three stars, HD 28843 (He weak), HD 182255 (He weak) and HD 223640 (B9SiSrCr), show a clear anti-phase correlation. The equivalent width of the HeI $5876 \AA$ line varies in phase with the photometric variations for the stars HD 43819 (B9 Si), HD 171247 (B8Si) and HD 176582 (He weak). As to HD 26571 the error on $\Phi$ is too large to draw any conclusion. In the case of HD 177003 (B3 He) nothing can be said since the Hipparcos light curve has too large a dispersion and a low amplitude. Hence no unique correlation exists, and this fact is independent of the spectral types of both groups of stars, which are all in the B5-B9 range. This result confirms the one obtained by Catalano \& Leone (1996). In an attempt to clarify the nature of the correlation between light and helium lines variations, these authors compared the emerging fluxes of two atmosphere models with the same effective temperature $\left(T_{\text {eff }}=15000 \mathrm{~K}\right)$ and gravity $(\log g=4.0)$ but with different helium abundance. The models were computed by means of the ATLAS9 code (Kurucz 1993) and are characterized by solar and zero helium abundance. By comparing these fluxes, they found no observable magnitude differences and concluded that the photometric variations presented by helium weak stars cannot be entirely ascribed to the non homogeneous distribution of helium on the stellar surface.

Figure 9 shows the measured average value of the equivalent width of the HeI $5876 \AA$ line together with the theoretical behaviour computed in the NLTE approximation by Leone \& Lanzafame (1998) for solar composition stars. We conclude the helium abundance is not peculiar for the stars HD 36589, HD 43819, HD 171247, HD 177003, HD 182255 and HD 209515, while helium is underabundant in the remaining stars. It is worthy note, that the equivalent width of the HeI5876 $\AA$ line for HD 182255 is close to the value of a solar composition main sequence star, even though this star is classified as an helium weak star.

Acknowledgements. This research has made use of the Simbad database, operated at CDS, Strasbourg, France. We would like to thank the referee, Dr. P. Martinez, for his useful suggestions that improved this paper.

\section{References}

Adelman S.J., 1997, A\&AS 127, 421

Adelman S.J., Knox jr. J.R., 1994, A\&AS 103, 1

Adelman S.J., Philip A.G.D., Adelman C.J., 1996, MNRAS 282,953

Babcock H.W., 1958, ApJS 3, 141

Bartholdy P., 1988, in: Halbwachs J.-L., Jasniewicz G., Egret D. (eds.), Détection et classification des étoiles variables. Comptes Rendus des Journées de Strasbourg, $10^{\mathrm{e}}$ réunion, 21 April 1988, p. 77

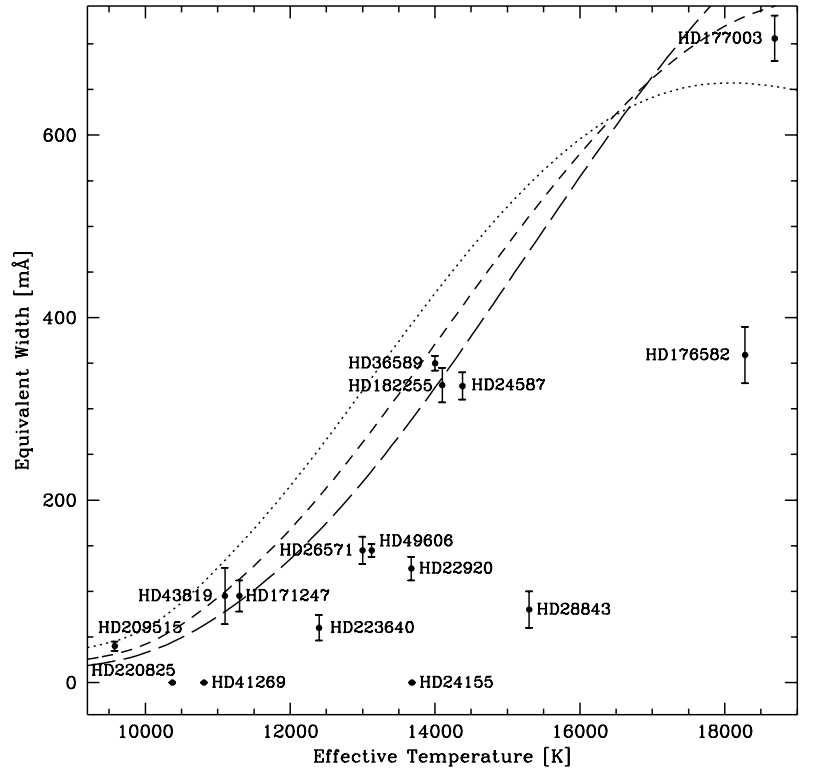

Fig. 9. Behaviour of equivalent width versus effective temperature for our sample of CP stars. Points represent the observations, errorbar extend by $1 \sigma$. Curves represent the NLTE calculations by Leone \& Lanzafame (1998) for log $g: 3.5$ (long dash), 4.0 (short dash) and 4.5 (dot)

Bond H.E., 1972, PASP 84, 446

Borra E.F., Landstreet J.D., 1980, ApJS 42, 421

Bossi M., Guerrero G., 1989, IBVS 3326

Catalano F.A., Leone F., 1996, A\&A 311, 230

Catalano F.A., Renson P., 1984, A\&AS 55, 371

Catalano F.A., Renson P., 1988, A\&AS 72, 1

Catalano F.A., Renson P., 1997, A\&AS 121, 57

Catalano F.A., Renson P., Leone F., 1991, A\&AS 86, 59

Catalano F.A., Renson P., Leone F., 1993, A\&AS 98, 269

Chunakova N.M., Bychov V.D., Glagolevskii Yu.V., 1981, Soobshch. Spetsial'noi Astrofiz. Obs. 31, 5

Cousins A.W.J., Stoy R.H., 1966, R. Obs. Bull. No. 121

Cowley A., 1972, AJ 77, 750

Cowley A., Cowley C., Jaschek M., Jaschek C., 1969, AJ 74, 375

Davis R.J., 1977, ApJ 213, 105

Dean J.F., 1980, Mon. Notes Astron. Soc. S. Afr. 39, 13

ESA SP-1200, 1997

Feinstein A., 1978, Rev. Mex. Astron. Astrofis. 2, 331

Glagolevskii Yu.V., Panov K., Chunakova N.M., 1985, Pis'ma AZh 11, 749

Gulliver A.F., 1971, Thesis, University of Toronto

Hao J.X., Huang L., Guo Z.H., 1996, A\&A 308, 499

Hauck B., North P., 1993, A\&A 269, 403

Horne J.H., Baliunas S.L., 1986, ApJ 302, 757

Hube D.P., Aikman G.C.L., 1991, PASP 103, 49

Hubrig S., Launhardt R., 1993, Line Variability in 33 Gem? In: Dworetsky M.M., Castelli F., Faraggiana R. (eds.) Proc. IAU Coll. 138, Peculiar versus Normal Phenomena in AType and Related Stars. ASP Conf. Ser. 44, 350

Jaschek M., Jaschek C., Arnal M., 1969, PASP 81, 650

Kurucz R.L., 1993, A new opacity-sampling model atmosphere program for arbitrary abundances. In: Dworetsky M.M., Castelli F., Castelli R. (eds.) IAU Col. 138, Peculiar versus 
normal phenomena in A-type and related stars. ASP Conf. Ser. 44,87

Leone F., Catanzaro G., 1998, A\&A 331, 627

Leone F., Lanzafame A.C., 1998, A\&A 330, 306

Leone F., Lanzafame A.C., Pasquini L., 1995, A\&A 293, 457

Lopez-Garcia Z., Adelman S.J., 1994, A\&AS 107, 353

Maitzen H.M., 1976, A\&A 51, 223

Maitzen H.M., 1980, IBVS 1735

Manfroid J., Mathys G., Cousins A.W.J., 1984, IBVS 2625

Manfroid J., Sterken C., Cunow B., et al., 1994, Third Catalogue of Stars Measured in the Long-Term Photometry of Variable Project (1990-1992), ESO Scientific Report No. 14

Mathys G., Manfroid J., 1985, A\&AS 60, 17

Mathys G., Manfroid J., Renson P., 1986, A\&AS 63, 403

Megessier C., 1974, A\&A 34, 53

Megessier C., 1975, A\&A 39, 263

Megessier C., Garnier R., 1972, Astrophys. Lett. 11, 113

Moon T.T., 1985, in: Communications from the University of London Observatory, No. 78

Moon T.T., Dworetsky M.M., 1985, MNRAS 217, 305

Morrison N.D., Wolff S.C., 1971, PASP 83, 474

Napiwotzki R., Schönberner D., Wenske V., 1993, A\&A 268, 653

North P., 1984, A\&AS 55, 259

North P., 1992, Photometric Periods of Some Old Si Stars. In: Glagolevskii Yu.V., Romanyuk I.I. (eds.) Proc. Int. Meeting on the Problem Physics and Evolution of the Stars. Stellar Magnetism. NAUKA, Sankt-Petersburg, p. 73
North P., Brown D.N., Landstreet J.T., 1992, A\&A 258, 389 Osawa K., 1965, Ann. Tokyo Astron. Obs. Ser. 2, 9, 123

Pedersen H., 1976, A\&A 49, 217

Pedersen H., 1979, A\&AS 35, 313

Pedersen H., Thomsen B., 1977, A\&AS 30, 11

Rakosch K. D., 1962, Lowell Obs. Bull. 5, 227

Renson P., Manfroid J., 1981, A\&AS 44, 23

Renson P., Gerbaldi M., Catalano F.A., 1991, A\&AS 89, 429

Ryabchicova T.A., Pavlova V.M., Davydova E.S., Piskunov N.E., 1996, Astron. Lett. 22, 822

Schöneich W., Zelwanowa E., 1984, In: Magnetic Stars Khokhlova V., et al., (ed.) Proceedings of the 6-th Conference on "Physics and Evolution of Stars", Riga, April 10-12 1984, p. 73

Sterken C., Manfroid J., Beele D., et al., 1995, Fourth Catalogue of Stars Measured in the Long-Term Photometry of Variable Project (1992-1994), ESO Scientific Report, No. 16

Stibbs D.W.N., 1950, MNRAS 110, 395

Strauss F.M., Ducati J.R., 1981, Revised Catalogue of Stellar Rotational Velocities

Vetö B., 1993, Do Bp Stars have "Flares"? In: Dworetsky M.M., Castelli F., Faraggiana R. (eds.) Proc. IAU Coll. 138, Peculiar versus Normal Phenomena in A-Type and Related Stars. ASP Conf. Ser. 44, 340

Waelkens C., 1985, A\&AS 61, 127

Winzer J.E., 1974, Thesis, University of Toronto

Wolff S.C., Preston G.W., 1978 ApJS 37, 371 